\title{
ALP dark matter in a primordial black hole dominated universe
}

\author{
Nicolás Bernal๑, ${ }^{1, *}$ Yuber F. Perez-Gonzalez ${ }^{2,3,4,5, \uparrow}$ Yong Xu๑, ${ }^{6, \$}$ and Óscar Zapata $\odot^{7,8}$ \\ ${ }^{1}$ Centro de Investigaciones, Universidad Antonio Nariño, Carrera 3 este \# 47A-15, Bogotá 110231, Colombia \\ ${ }^{2}$ Theoretical Physics Department, Fermi National Accelerator Laboratory, \\ P.O. Box 500, Batavia, Illinois 60510, USA \\ ${ }^{3}$ Department of Physics and Astronomy, Northwestern University, Evanston, Illinois 60208, USA \\ ${ }^{4}$ Colegio de Fsica Fundamental e Interdisciplinaria de las Amricas (COFI), \\ 254 Norzagaray Street, San Juan, Puerto Rico 00901 \\ ${ }^{5}$ Institute for Particle Physics Phenomenology, Durham University, South Road, Durham DH1 3LE, \\ United Kingdom \\ ${ }^{6}$ Bethe Center for Theoretical Physics and Physikalisches Institut, Universität Bonn, \\ Nussallee 12, 53115 Bonn, Germany \\ ${ }^{7}$ Instituto de Física, Universidad de Antioquia, \\ Calle 70 \# 52-21, Apartado Aéreo 1226, Medellín, Colombia
}

(Received 15 October 2021; accepted 16 November 2021; published 16 December 2021)

\begin{abstract}
We investigate the phenomenological consequences of axionlike particle (ALP) dark matter with an early matter domination triggered by primordial black holes (PBHs). We focus on light BHs with masses smaller than $\sim 10^{9} \mathrm{~g}$ which fully evaporate before big bang nucleosynthesis. We numerically solve the coupled Boltzmann equations, carefully taking the graybody factors and $\mathrm{BH}$ angular momentum into account. We find that the entropy injection from PBH evaporation dilutes the ALP relic abundance originally produced via the vacuum misalignment mechanism, opening the parameter space with larger scales $f_{a}$ or, equivalently, smaller ALP-photon couplings $g_{a \gamma}$, within the reach of future detectors as ABRACADABRA, KLASH, ADMX, and DM-Radio. Moreover, the ALP minicluster masses can be several orders of magnitude larger if the early universe features an $\mathrm{PBH}$ dominated epoch. For the relativistic ALPs produced directly from Hawking radiation, we find that their contribution to the dark radiation is within the sensitivity of next generation cosmic microwave background experiments. For the sake of completeness, we also revisit the particular case of the QCD axion.
\end{abstract}

DOI: $10.1103 /$ PhysRevD.104.123536

\section{INTRODUCTION}

Primordial black holes (PBHs), which could have been copiously produced in the early universe due to large density fluctuations, are attracting intensive investigations; for recent reviews see, e.g., Refs. [1-4]. Those with masses larger than $\sim 10^{15} \mathrm{~g}$ have not fully evaporated at present and are potential cold dark matter (DM) candidates. On the contrary, light PBHs could also have been created, and have a not less interesting phenomenology. For example, in their evaporation process they can source particle DM or dark

\footnotetext{
"nicolas.bernal@uan.edu.co

†uber.f.perez-gonzalez@durham.ac.uk

*yongxu@th.physik.uni-bonn.de

§oalberto.zapata@udea.edu.co
}

Published by the American Physical Society under the terms of the Creative Commons Attribution 4.0 International license. Further distribution of this work must maintain attribution to the author(s) and the published article's title, journal citation, and DOI. Funded by SCOAP . radiation (DR) [5-17], trigger baryogenesis [18-22], and radiate all new degrees of freedom, such as right-handed neutrinos [23]. Along the same research direction, in this work we focus on phenomenology of QCD axion and axionlike particles (ALPs) in a universe dominated by PBHs lighter than $\sim 10^{9} \mathrm{~g},{ }^{1}$ which have fully evaporated before big bang nucleosynthesis (BBN).

The QCD axion [25,26], a pseudo-Nambu-Goldstone boson resulting from the spontaneous breaking of the global $U(1)$ Peccei-Quinn symmetry [27-29], is a wellmotivated candidate for DM. In the early universe, it can be produced via a number of processes, being the vacuum misalignment mechanism [30-32] and the decay of topological defects [33] the most popular in the literature. In the standard cosmological scenario, the axion window is rather narrow; in particular, an axion with mass $m_{a} \simeq$ $\left[10^{-6}-10^{-5}\right] \mathrm{eV}$ (or correspondingly to a Peccei-Quinn

${ }^{1}$ For production mechanism of such light PBHs, see, e.g., Ref. [24]. 
scale $f_{a} \simeq 10^{12} \mathrm{GeV}$ ) is expected, if one does not want to introduce fine-tuning of the initial misalignment angle $\theta_{i}$ [34-36]. ${ }^{2}$ In light of the accumulated interesting implications for PBHs [39-44], it has recently been shown that the axion DM window can be enlarged to a mass as low as $m_{a} \sim \mathcal{O}\left(10^{-8}\right) \mathrm{eV}$ if the universe features an early PBHs dominated epoch [45].

In this paper, we go beyond the QCD axion DM and consider general ALPs (see, e.g., Refs. [34,35,46-49]). ALPs could also arise from the spontaneous breaking of a global $U$ (1) symmetry, similar to the QCD axion. They are also quite ubiquitous in string theory [50,51]. Different from QCD axions, ALPs do not solve the strong $C P$ problem since they are in general not involved in the strong interaction. However, they serve as a good candidate for DM (see, e.g., Ref. [48]). In this paper, we focus on ALP DM generated via the usual misalignment mechanism. Therefore, our results are complementary to those presented in Ref. [44]. In the standard cosmological scenario, the ALP relic density depends on three parameters: the decay constant $f_{a}$, its mass $m_{a}$, and the initial misalignment angle $\theta_{i}$, leading to strong bounds on the viable parameter space for natural values of $\theta_{i}$. However, new regions of the parameter space become viable with a nonstandard cosmological epoch before BBN. We note that the phenomenology of ALPs as DM with nonstandard cosmological epochs such as kination or an early matter phase was investigated in Ref. [52] recently, where it was shown that a broader parameter is available and could be within the reach of several proposed experiments. Similar to Ref. [45] and different from Ref. [52], we focus on the phenomenological consequences of ALPs in an early matter epoch triggered by PBHs.

Since PBHs effectively behave as matter, with an energy density that redshifts slower than radiation, it could be expected that the early universe has undergone a $\mathrm{PBH}$ dominated epoch. Moreover, it is worthwhile to mention some differences between an early matter dominated epoch triggered by PBHs and by a long-lived heavy particle. In the former case, the evaporation (or decay) rate is time dependent while the latter is not. Besides, an early $\mathrm{PBH}$ era would inevitably give rise to gravitational wave (GW) signatures [53-57], offering an interesting avenue to constrain on the initial amount of energy density in the early matter epoch, while such kinds of constraints are absent in the case of a heavy particle.

For the case with an early PBH era, we numerically solve a system of coupled Boltzmann equations for the background [PBHs and standard model (SM) radiation] based on the code developed in Refs. $[15,16]$, carefully including the graybody factor in PBHs spectra, and considering the effect of the PBH angular momentum. We find an increased

\footnotetext{
${ }^{2}$ We note that for very low scale inflation, the axion field follows a Bunch-Davies distribution, in which case $\theta_{i}$ could be small $[37,38]$.
}

allowed parameter space with larger $f_{a}$ or, equivalently, smaller ALP-photon coupling $g_{a \gamma}$ due to the entropy injection from $\mathrm{PBH}$ evaporation. Furthermore, in the scenario where the Peccei-Quinn symmetry is spontaneously broken after inflation, the gravitational clump of ALP (axion) density inhomogeneities at the time of the matterradiation equality gives rise to miniclusters [58-62]. Due to the PBH domination epoch, miniclusters with larger masses could be formed.

Additionally, ALPs and axions are directly radiated from PBHs evaporation, being ultrarelativistic, and thus contributing to the DR. Taking carefully into account the effect of the $\mathrm{PBH}$ spin, we numerically compute the contribution to $\Delta N_{\text {eff }}$, which is within the sensitivity of next generation cosmic microwave background (CMB) experiments [63-66]. We also find that for Kerr PBHs, $\Delta N_{\text {eff }}$ turns out to be smaller than for nonrotating Schwarzschild PBHs.

The paper is organized as follows. We first revisit PBH evaporation for both Schwarzschild and Kerr BHs in Sec. II. In Sec. III, we estimate the background evolution by setting up the coupled Boltzmann equations and the formalism for estimating the entropy injection. Sec. IV is devoted to the phenomenological consequences of ALP DM. For the sake of completeness, in Sec. V we revisit QCD axion DM. Additionally, in Sec. VI we focus on the axion and ALP DR directly produced from $\mathrm{PBH}$ evaporation. Finally, we sum up our findings in Sec. VII. We use natural units where $\hbar=$ $c=k_{\mathrm{B}}=1$ throughout this manuscript.

\section{PRIMORDIAL BLACK HOLE EVAPORATION}

PBHs are hypothetical objects which could be generated due to inhomogeneities of density perturbations in the early universe $[67,68]$. When these fluctuations reenter the horizon, if above a threshold, they could collapse and form a $\mathrm{BH}$ according to the Press-Schechter formalism [69]. In this paper, we focus on the case where PBHs form in a radiation-dominated epoch. With an initial cosmic temperature $T=T_{\text {in }}$, the initial PBH mass is given by the whole mass within the particle horizon $[1,70]$,

$$
M_{\mathrm{in}} \equiv M_{\mathrm{BH}}\left(T_{\mathrm{in}}\right)=\frac{4 \pi}{3} \gamma \frac{\rho_{R}\left(T_{\mathrm{in}}\right)}{H^{3}\left(T_{\mathrm{in}}\right)},
$$

where we take the efficiency factor to be $\gamma \simeq 0.2$. Additionally, $\rho_{R}$ and $H$ correspond to the SM energy density and the Hubble expansion rate, respectively. For simplicity, we assume a monochromatic mass spectrum, such that all PBHs were created with the same mass. The extension to more realistic mass distributions will be considered elsewhere. The PBH initial energy density $\rho_{\mathrm{BH}}\left(T_{\text {in }}\right)$ is usually related to the SM radiation energy density at formation via the $\beta$ parameter 


$$
\beta \equiv \frac{\rho_{\mathrm{BH}}\left(T_{\mathrm{in}}\right)}{\rho_{R}\left(T_{\mathrm{in}}\right)}
$$

Since $\rho_{\mathrm{BH}}$ redshifts slower than radiation, an early PBHdominated epoch with $\rho_{\mathrm{BH}}>\rho_{R}$ is triggered if $\beta>\beta_{c}$, with

$$
\beta_{c} \equiv \frac{T_{\mathrm{ev}}}{T_{\mathrm{in}}}
$$

where $T_{\mathrm{ev}}$, given by [45]

$$
T_{\mathrm{ev}} \simeq\left(\frac{g_{\star}\left(T_{\mathrm{in}}\right)}{640}\right)^{1 / 4}\left(\frac{M_{P}^{5}}{M_{\mathrm{in}}^{3}}\right)^{1 / 2},
$$

is the SM temperature at which PBHs completely evaporate. Additionally, $M_{P} \equiv 1 / \sqrt{8 \pi G}$ denotes the reduced Planck mass, and $g_{\star}(T)$ corresponds to the number of relativistic degrees of freedom contributing to the SM energy density. It is interesting to note that if $\beta>\beta_{c}$, PBHs start to dominate the total energy density of the universe at $T=T_{\text {eq }}$, defined as $\rho_{R}\left(T_{\text {eq }}\right)=\rho_{\mathrm{BH}}\left(T_{\text {eq }}\right)$, with

$$
T_{\text {eq }}=\beta T_{\text {in }}\left(\frac{g_{\star s}\left(T_{\text {in }}\right)}{g_{\star s}\left(T_{\text {eq }}\right)}\right)^{1 / 3},
$$

with $g_{\star s}(T)$ being the number of relativistic degrees of freedom contributing to the SM entropy.

Several bounds exist in the PBH parameter space spanned by the initial fraction $\beta$ and mass $M_{\text {in }}$ [1,2]. Here, we will focus on $\mathrm{PBHs}$ that evaporated before $\mathrm{BBN}$, thus having masses smaller than $\sim 10^{9} \mathrm{~g}$. Although there exist constraints on such light PBHs, they are typically model dependent $[1,2,23]$. Nevertheless, recent constraints have been derived after considering the GWs emitted from the Hawking evaporation. In particular, a backreaction problem can be avoided if the energy contained in GWs never overtakes the one of the background universe [54]. More importantly, a modification of BBN predictions due to the energy density stored in GWs can be avoided if [55]

$$
\beta \lesssim 1.1 \times 10^{-6}\left(\frac{\gamma}{0.2}\right)^{-\frac{1}{2}}\left(\frac{M_{\text {in }}}{10^{4} \mathrm{~g}}\right)^{-\frac{17}{24}}
$$

Next, we briefly describe the time evolution properties of Schwarzschild and Kerr PBHs relevant for our purposes. Further details can be found in Refs. [15,16,71].

\section{A. Schwarzschild black holes}

For the simplest BH scenario, PBHs are only characterized by their mass, so that their horizon temperature $T_{\mathrm{BH}}$ is determined according to [67]

$$
T_{\mathrm{BH}}=\frac{M_{P}^{2}}{M_{\mathrm{BH}}} \simeq 10^{13} \mathrm{GeV}\left(\frac{1 \mathrm{~g}}{M_{\mathrm{BH}}}\right) .
$$

All particles with masses smaller than $T_{\mathrm{BH}}$ can be emitted via Hawking radiation. Within a time interval $d t$ and momentum $[p, p+d p]$, the energy spectrum of an emitted species $i$ with spin $s_{i}$, mass $\mu_{i}$, and internal degrees of freedom $g_{i}$ can be described as $[15,67]$

$$
\frac{\mathrm{d}^{2} \mathcal{N}_{i}}{\mathrm{~d} p \mathrm{~d} t}=\frac{g_{i}}{2 \pi^{2}} \frac{\sigma_{s_{i}}\left(M_{\mathrm{BH}}, \mu_{i}, p\right)}{\exp \left[E_{i}(p) / T_{\mathrm{BH}}\right]-(-1)^{2 s_{i}}} \frac{p^{3}}{E_{i}(p)},
$$

with $E_{i}(p)=\sqrt{p^{2}+\mu_{i}^{2}}$ denoting the energy and $\sigma_{s_{i}}$ describing the absorption cross section. Summing over all possible particle species and integrating over the phase space in Eq. (2.8), one can obtain the BH mass evolution, which is shown to be $[72,73]$

$$
\begin{aligned}
\left.\frac{\mathrm{d} M_{\mathrm{BH}}}{\mathrm{d} t} \equiv \sum_{i} \frac{\mathrm{d} M_{\mathrm{BH}}}{\mathrm{d} t}\right|_{i} & =-\sum_{i} \int_{0}^{\infty} E_{i} \frac{\mathrm{d}^{2} \mathcal{N}_{i}}{\mathrm{~d} p \mathrm{~d} t} \mathrm{~d} p \\
& =-\varepsilon\left(M_{\mathrm{BH}}\right) \frac{M_{P}^{4}}{M_{\mathrm{BH}}^{2}},
\end{aligned}
$$

with the mass evaporation function $\varepsilon\left(M_{\mathrm{BH}}\right) \equiv \sum_{i} g_{i} \varepsilon_{i}\left(z_{i}\right)$, where the contribution per degree of freedom $\varepsilon_{i}\left(z_{i}\right)$ is given by [15]

$$
\varepsilon_{i}\left(z_{i}\right)=\frac{27}{128 \pi^{3}} \int_{z_{i}}^{\infty} \frac{\psi_{s_{i}}\left(x, z_{i}\right)\left(x^{2}-z_{i}^{2}\right)}{\exp (x)-(-1)^{2 s_{i}}} x \mathrm{~d} x
$$

where $\quad x=E_{i} / T_{\mathrm{BH}}, \quad z_{i}=\mu_{i} / T_{\mathrm{BH}}, \quad$ and $\quad \psi_{s_{i}}(E, \mu) \equiv$ $\sigma_{s_{i}}(E, \mu) /\left(27 \pi G^{2} M_{\mathrm{BH}}^{2}\right)$.

\section{B. Kerr black holes}

For the case of BHs with a nonzero spin, the horizon temperature has an additional dependence on the spin parameter $a_{\star} \in[0,1]$ (with $a_{\star}=0$ corresponding to the Schwarzschild limit),

$$
T_{\mathrm{BH}}=\frac{2 M_{P}^{2}}{M_{\mathrm{BH}}} \frac{\sqrt{1-a_{\star}}}{1+\sqrt{1-a_{\star}}},
$$

where $a_{\star} \equiv 8 \pi J M_{P}^{2} / M_{\mathrm{BH}}^{2}$, with $J$ denoting the total $\mathrm{BH}$ angular momentum. The energy spectrum is modified by the presence of the angular momentum, introducing an explicit dependence on the total $l$ and axial $m$ angular quantum numbers,

$$
\frac{\mathrm{d}^{2} \mathcal{N}_{i}}{\mathrm{~d} p \mathrm{~d} t}=\frac{g_{i}}{2 \pi^{2}} \sum_{l=s_{i}} \sum_{m=-l}^{l} \frac{\sigma_{s_{i}}^{l m}\left(M_{\mathrm{BH}}, p, a_{\star}\right)}{\exp \left[\left(E_{i}-m \Omega\right) / T_{\mathrm{BH}}\right]-(-1)^{2 s_{i}}} \frac{p^{3}}{E_{i}},
$$


with $\Omega=\left(4 \pi a_{\star} M_{P}^{2} / M_{\mathrm{BH}}\right)\left(1+\sqrt{1-a_{\star}^{2}}\right)^{-1}$ horizon angular velocity. The time evolution for the $\mathrm{BH}$ spin and mass is described by the following set of equations [71]:

$$
\begin{array}{r}
\frac{d M_{\mathrm{BH}}}{d t}=-\varepsilon\left(M_{\mathrm{BH}}, a_{\star}\right) \frac{M_{P}^{4}}{M_{\mathrm{BH}}^{2}}, \\
\frac{d a_{\star}}{d t}=-a_{\star}\left[\gamma\left(M_{\mathrm{BH}}, a_{\star}\right)-2 \varepsilon\left(M_{\mathrm{BH}}, a_{\star}\right)\right] \frac{M_{P}^{4}}{M_{\mathrm{BH}}^{3}},
\end{array}
$$

where the evaporation functions $\varepsilon\left(M_{\mathrm{BH}}, a_{\star}\right)$ and $\gamma\left(M_{\mathrm{BH}}\right)$ are given by

$\varepsilon_{i}\left(z_{i}, a_{\star}\right)=\frac{27}{128 \pi^{3}} \int_{z_{i}}^{\infty} \sum_{l, m} \frac{\psi_{s_{i}}^{l m}\left(x, a_{\star}\right)\left(x^{2}-z_{i}^{2}\right)}{\exp \left(x^{\prime} / 2 f\left(a_{\star}\right)\right)-(-1)^{2 s_{i}}} x \mathrm{~d} x$,

$\gamma_{i}\left(z_{i}, a_{\star}\right)=\frac{27}{16 \pi^{2}} \int_{z_{i}}^{\infty} \sum_{l, m} \frac{m \psi_{s_{i}}^{l m}\left(x, a_{\star}\right)\left(x^{2}-z_{i}^{2}\right)}{\exp \left(x^{\prime} / 2 f\left(a_{\star}\right)\right)-(-1)^{2 s_{i}}} \mathrm{~d} x$,

with $\quad x^{\prime}=x-m \Omega^{\prime}, \quad$ being $^{3} \quad x=M_{\mathrm{BH}} E_{i} / M_{P}^{2}, \quad \Omega^{\prime}=$ $M_{\mathrm{BH}} \Omega / M_{P}^{2}$, and $f\left(a_{\star}\right)=\sqrt{1-a_{\star}} /\left(1+\sqrt{1-a_{\star}}\right)$. This set of time evolution equations are the basis for describing the effect of a PBH dominated universe on the ALP and axion DM genesis.

\section{BACKGROUND EVOLUTION}

The evolution of the SM entropy density $s(T)=$ $\frac{2 \pi^{2}}{45} g_{\star s}(T) T^{3}$ can be tracked via the Boltzmann equation

$$
\frac{d s}{d t}+3 H s=-\left.\frac{1}{T} \frac{\rho_{\mathrm{BH}}}{M_{\mathrm{BH}}} \frac{d M_{\mathrm{BH}}}{d t}\right|_{\mathrm{SM}},
$$

where $\quad H^{2}=\left(\rho_{R}+\rho_{\mathrm{BH}}\right) /\left(3 M_{P}^{2}\right)$, with $\quad \rho_{R}(T)=$ $\frac{\pi^{2}}{30} g_{\star}(T) T^{4}$, and $\left.\frac{-1}{M_{\mathrm{BH}}} \frac{d M_{\mathrm{BH}}}{d t}\right|_{\mathrm{SM}}$ corresponds to the timedependent $\mathrm{PBH}$ evaporation rate into SM particles. We emphasize that Eq. (3.1) has to be numerically solved together with Eq. (2.9) in the case of a Schwarzschild PBH, or with Eqs. (2.13) in the case of a Kerr PBH, to extract the dynamics of the background, and in particular the evolution of the SM temperature and the (nonconserved) SM entropy.

In a SM radiation dominated scenario, the Hubble expansion rate takes the simple form

$$
H(T)=H_{R}(T) \equiv \sqrt{\frac{\rho_{R}(T)}{3 M_{P}^{2}}}=\frac{\pi}{3} \sqrt{\frac{g_{\star}(T)}{10}} \frac{T^{2}}{M_{P}} .
$$

However, PBHs can have a strong impact on the evolution of the background dynamics [74]. With an early PBH dominated epoch, cosmology can be characterized by four distinct regimes, where the Hubble expansion rate is given by [45]

$$
H(T) \simeq \begin{cases}H_{R}(T) & \text { for } T \geq T_{\mathrm{eq}}, \\ H_{R}\left(T_{\mathrm{eq}}\right)\left[\frac{g_{\star s}(T)}{g_{\star s}\left(T_{\mathrm{eq}}\right)}\left(\frac{T}{T_{\mathrm{eq}}}\right)^{3}\right]^{1 / 2} & \text { for } T_{\mathrm{eq}} \geq T \geq T_{\mathrm{c}}, \\ H_{R}\left(T_{\mathrm{ev}}\right)\left[1-\frac{720}{\pi g_{\star}\left(T_{\mathrm{in}}\right)} \frac{M_{\mathrm{in}}^{3}}{M_{P}^{4}} \frac{H_{R}^{2}\left(T_{\mathrm{ev}}\right)-H_{R}^{2}(T)}{H_{R}\left(T_{\mathrm{ev}}\right)}\right] & \text { for } T_{\mathrm{c}} \geq T \geq T_{\mathrm{ev}}, \\ H_{R}(T) & \text { for } T_{\mathrm{ev}} \geq T,\end{cases}
$$

which can be simplified to

$$
H(T) \simeq \begin{cases}H_{R}(T) & \text { for } T \geq T_{\mathrm{eq}}, \\ H_{R}\left(T_{\mathrm{eq}}\right)\left(\frac{T}{T_{\mathrm{eq}}}\right)^{3 / 2} & \text { for } T_{\mathrm{eq}} \gg T \gg T_{\mathrm{c}}, \\ H_{R}\left(T_{\mathrm{ev}}\right)\left(\frac{T}{T_{\mathrm{ev}}}\right)^{4} & \text { for } T_{\mathrm{c}} \gg T \gg T_{\mathrm{ev}}, \\ H_{R}(T) & \text { for } T_{\mathrm{ev}} \geq T,\end{cases}
$$

${ }^{3}$ Note that the definition of $x$ is the same as in the Schwarzschild case. for being more conveniently used in the next expressions. Here, we have introduced the temperature scale $T_{\mathrm{c}}$, from which the SM radiation does not scale as free radiation (i.e., $\rho_{R}(R) \propto R^{-1}$ with $R$ being the scale factor) due to the entropy injected by the PBH evaporation. It is given by [45]

$$
T_{\mathrm{c}} \simeq\left[\frac{g_{\star}\left(T_{\mathrm{in}}\right) \pi}{5760} \frac{M_{P}^{10} T_{\mathrm{eq}}}{M_{\mathrm{in}}^{6}}\right]^{1 / 5} \simeq\left(T_{\mathrm{eq}} T_{\mathrm{ev}}^{4}\right)^{1 / 5} .
$$

Additionally, during its evaporation, $\mathrm{PBHs}$ radiate SM particles and therefore dilute all previously produced species. The entropy injection factor is [45] 


$$
\frac{S(T)}{S\left(T_{\mathrm{ev}}\right)} \simeq \begin{cases}\frac{g_{\star s}\left(T_{\mathrm{eq}}\right)}{g_{\star s}\left(T_{\mathrm{ev}}\right)} \frac{g_{\star}\left(T_{\mathrm{ev}}\right)}{g_{\star}\left(T_{\mathrm{eq}}\right)} \frac{T_{\mathrm{ev}}}{T_{\mathrm{eq}}} & \text { for } T \geq T_{\mathrm{c}}, \\ \frac{g_{\star s}(T)}{g_{\star s}\left(T_{\mathrm{ev}}\right)}\left(\frac{T}{T_{\mathrm{ev}}}\right)^{3}\left[1-\frac{720}{\pi g_{\star}\left(T_{\mathrm{in}}\right)} \frac{M_{\mathrm{in}}^{3}}{M_{P}^{4}} \frac{H_{R}^{2}\left(T_{\mathrm{ev}}\right)-H_{R}^{2}(T)}{H_{R}\left(T_{\mathrm{ev}}\right)}\right]^{-2} & \text { for } T_{\mathrm{c}} \geq T \geq T_{\mathrm{ev}}, \\ 1 & \text { for } T_{\mathrm{ev}} \geq T,\end{cases}
$$

which can be simplified to

$$
\frac{S(T)}{S\left(T_{\mathrm{ev}}\right)} \simeq \begin{cases}\frac{T_{\mathrm{ev}}}{T_{\mathrm{eq}}} & \text { for } T \geq T_{\mathrm{c}} \\ \left(\frac{T_{\mathrm{ev}}}{T}\right)^{5} & \text { for } T_{\mathrm{c}} \geq T \geq T_{\mathrm{ev}} \\ 1 & \text { for } T_{\mathrm{ev}} \geq T\end{cases}
$$

again to ease further analytical estimations. We note that in Eqs. (3.6) and (3.7) there are three distinctive regimes, and not four as in Eq. (3.3).

Finally, it is important to emphasize that all these analytical estimations help to understand the dynamics of the cosmological evolution. However, hereafter full numerical solutions of the background will be used. In our code, we solve such Boltzmann equations together with the time evolution equations for the PBHs, given in Eqs. (2.13). Notice that taking as the initial value $a_{\star}=0$, the evolution equations in (2.13) reduce to the ones for the Schwarzschild scenario, i.e., Eq. (2.9). In other words, such a scenario is included in the more general Kerr case. Thus, we take in general the equations for the Kerr case, putting $a_{\star}=0$ whenever we talk about Schwarzschild PBHs. Besides, in the evaporation functions $\varepsilon\left(M_{\mathrm{BH}}, a_{\star}\right)$ and $\gamma\left(M_{\mathrm{BH}}\right)$ we have included an additional, almost massless, pseudoscalar degree of freedom, corresponding to the ALP or the axion.

\section{ALP DARK MATTER}

In this section, we focus on a general light pseudoscalar, namely the ALP [46-48]. Similar to the QCD axion, ALPs could arise as a consequence of the spontaneous breaking of a global $U(1)$ symmetry or, alternatively, they could emerge from string theory [50,51].

In the so-called misalignment mechanism, the present energy density stored in the zero mode of an ALP field $a$ of mass $m_{a}$ can be obtained by solving the equation of motion [30-32,75]

$$
\ddot{\theta}+3 H(t) \dot{\theta}+m_{a}^{2} \sin \theta=0,
$$

where $\theta(t) \equiv a(t) / f_{a}$ and $f_{a}$ is the Peccei-Quinn (PQ) symmetry breaking energy scale. In this scenario, the ALP field starts to roll about the minimum of the potential once the Hubble friction is overcome by the potential term. Coherent oscillations of the ALPs are set around the temperature $T_{\text {osc }}$ defined as

$$
3 H\left(T_{\text {osc }}\right) \equiv m_{a}
$$

In the standard cosmological scenario, as the SM entropy is conserved, the ALP energy density $\rho_{a}$ at present is given by

$$
\rho_{a}\left(T_{0}\right)=\rho_{a}\left(T_{\mathrm{osc}}\right) \frac{s\left(T_{0}\right)}{s\left(T_{\mathrm{osc}}\right)},
$$

where $T_{0}$ is the CMB temperature at present, and we considered the conservation of the ALP number density in a comoving volume. Additionally, $\rho_{a}\left(T_{\text {osc }}\right) \simeq \frac{1}{2} m_{a}^{2} f_{a}^{2} \theta_{i}^{2}$ in the limit in which the kinetic energy is neglected, and for a quadratic potential. Moreover, $\theta_{i}$ denotes the initial misalignment angle. The entropy injection from PBHs evaporation would inevitably dilute the energy density so that one has

$$
\begin{aligned}
\rho_{a}\left(T_{0}\right) & =\rho_{a}\left(T_{\mathrm{osc}}\right) \frac{s\left(T_{0}\right)}{s\left(T_{\mathrm{osc}}\right)} \times \frac{S\left(T_{\mathrm{osc}}\right)}{S\left(T_{\mathrm{ev}}\right)} \\
& \simeq \frac{1}{2} m_{a}^{2} f_{a}^{2} \theta_{i}^{2} \frac{s\left(T_{0}\right)}{s\left(T_{\mathrm{osc}}\right)} \times \frac{S\left(T_{\mathrm{osc}}\right)}{S\left(T_{\mathrm{ev}}\right)},
\end{aligned}
$$

where $S(T)=s(T) R^{3}$ corresponds to the total SM entropy. With such an energy density, one can compute the ALP DM relic density via

$$
\Omega_{a} h^{2} \equiv \frac{\rho_{a}\left(T_{0}\right)}{\rho_{c} / h^{2}},
$$

where $\rho_{c} / h^{2} \simeq 1.1 \times 10^{-5} \mathrm{GeV} / \mathrm{cm}^{3}$ is the critical energy density and $s\left(T_{0}\right) \simeq 2.69 \times 10^{3} \mathrm{~cm}^{-3}$ is the entropy density at present, which should match the total DM abundance $\Omega h^{2} \simeq 0.12[76]$.

Knowing the Hubble expansion rate given in the previous section, the ALP oscillation temperature $T_{\text {osc }}$ can be estimated to be

$$
T_{\mathrm{osc}} \simeq \begin{cases}\left(\frac{1}{\pi} \sqrt{\frac{10}{g_{\star}}} M_{P} m_{a}\right)^{1 / 2} & \text { for } T \geq T_{\mathrm{eq}}, \\ \left(\frac{1}{\pi} \sqrt{\frac{10}{g_{\star}}} \frac{M_{P} m_{a}}{\sqrt{T_{\mathrm{eq}}}}\right)^{2 / 3} & \text { for } T_{\mathrm{eq}} \geq T \geq T_{\mathrm{c}}, \\ \left(\frac{1}{\pi} \sqrt{\frac{10}{g_{\star}}} M_{P} m_{a} T_{\mathrm{ev}}^{2}\right)^{1 / 4} & \text { for } T_{\mathrm{c}} \geq T \geq T_{\mathrm{ev}}, \\ \left(\frac{1}{\pi} \sqrt{\frac{10}{g_{\star}}} M_{P} m_{a}\right)^{1 / 2} & \text { for } T_{\mathrm{ev}} \geq T .\end{cases}
$$

Using Eq. (4.4), one can compute the relic density in the aforementioned four regimes, which becomes 


$$
\Omega_{a} h^{2} \simeq \frac{45 \theta_{i}^{2}}{4 \pi^{2} g_{\star s}} \frac{s\left(T_{0}\right)}{\rho_{c} / h^{2}} \times \begin{cases}\left(\sqrt{\frac{g_{\star}}{10}} \frac{\pi}{M_{P}}\right)^{3 / 2} \frac{T_{\mathrm{ev}}}{T_{\mathrm{eq}}} m_{a}^{1 / 2} f_{a}^{2} & \text { for } T_{\mathrm{osc}} \geq T_{\mathrm{eq}}, \\ \left(\sqrt{\frac{g_{\star}}{10}} \frac{\pi}{M_{P}}\right)^{2} T_{\mathrm{ev}} f_{a}^{2} & \text { for } T_{\mathrm{eq}} \geq T_{\mathrm{osc}} \geq T_{\mathrm{ev}}, \\ \left(\sqrt{\frac{g_{\star}}{10}} \frac{\pi}{M_{P}}\right)^{3 / 2} m_{a}^{1 / 2} f_{a}^{2} & \text { for } T_{\mathrm{ev}} \geq T_{\mathrm{osc}}\end{cases}
$$

or equivalently

$$
\frac{\Omega_{a} h^{2}}{0.12} \simeq \begin{cases}\left(\frac{\theta_{i}}{0.5}\right)^{2}\left(\frac{T_{\mathrm{ev}}}{4 \mathrm{MeV}}\right)\left(\frac{81 \mathrm{GeV}}{T_{\mathrm{eq}}}\right)\left(\frac{m_{a}}{10^{-3} \mathrm{eV}}\right)^{1 / 2}\left(\frac{f_{a}}{5 \times 10^{14} \mathrm{GeV}}\right)^{2} & \text { for } T_{\mathrm{osc}} \geq T_{\mathrm{eq}} \\ \left(\frac{\theta_{i}}{0.5}\right)^{2}\left(\frac{T_{\mathrm{ev}}}{4 \mathrm{MeV}}\right)\left(\frac{f_{a}}{10^{15} \mathrm{GeV}}\right)^{2} & \text { for } T_{\mathrm{eq}} \geq T_{\mathrm{osc}} \geq T_{\mathrm{ev}} \\ \left(\frac{\theta_{i}}{0.5}\right)^{2}\left(\frac{m_{a}}{10^{-15} \mathrm{eV}}\right)^{1 / 2}\left(\frac{f_{a}}{3 \times 10^{15} \mathrm{GeV}}\right)^{2} & \text { for } T_{\mathrm{ev}} \geq T_{\mathrm{osc}}\end{cases}
$$

Note that the expressions for $\Omega_{a} h^{2}$ are identical for $T_{\text {eq }} \geq$ $T_{\mathrm{osc}} \geq T_{\mathrm{c}}$ and $T_{\mathrm{c}} \geq T_{\mathrm{ev}}$. Considering the full numerical solution of the Boltzmann equations, we present in Fig. 1 the parameter space generating the whole observed ALP DM abundance for benchmark values of $m_{a}=10^{-7} \mathrm{eV}$ and $f_{a}=10^{14} \mathrm{GeV}$, while assuming Schwarzschild ( $a_{\star}=0$, red band) or Kerr $\left(a_{\star}=0.999\right.$, blue band) PBHs. The thickness of the bands corresponds to an initial misalignment angle $0.5 \leq \theta_{i} \leq \pi / \sqrt{3}{ }^{4}$. The regions on the right (left) of the bands produce a DM underabundance (overabundance). Additionally, the gray regions are in tension with $\mathrm{BBN}\left(T_{\mathrm{ev}}<4 \mathrm{MeV}\right.$ [77-82]), whereas the green region with GWs [i.e., Eq. (2.6)]. Finally, the dashed red line corresponds to $\beta=\beta_{c}$, limiting the region where $\mathrm{PBH}$ energy density is subdominant with respect to SM radiation, and therefore one has a standard cosmological scenario (below), with the region where a nonstandard cosmological expansion triggered by PBHs occurs (above). It is important to emphasize that these results were obtained using the numerical code developed in Refs. [15,16], including the additional ALP degree of freedom. We have checked that the numerical estimations in Eq. (4.7) fit well the analytical results.

As seen in Fig. 1, the presence of an era dominated by PBHs can have a strong impact on the ALP production in the early universe. The maximal deviation from the standard cosmological scenario corresponds to long-lived PBHs evaporating just before the onset of BBN, and the maximal value for $\beta$ allowed by GWs. This happens for $M_{\text {in }} \simeq 5.7 \times 10^{8} \mathrm{~g}$ and $\beta \simeq 4.6 \times 10^{-10}$ for $a_{\star}=0$, or $M_{\text {in }} \simeq 7.6 \times 10^{8} \mathrm{~g}$ and $\beta \simeq 3.5 \times 10^{-10}$ for $a_{\star}=0.999$, and corresponds to the upper right white corners in Fig. 1. This maximal deviation from the standard

\footnotetext{
${ }^{4}$ The lower bound comes from assuming an $\mathcal{O}(1)$ misalignment angle that avoids introducing fine-tuning of the initial conditions, whereas the upper bound is an effective average misalignment angle, typical from postinflationary scenarios.
}

cosmological scenario is shown in Fig. 2, for the benchmark $M_{\text {in }} \simeq 5.7 \times 10^{8} \mathrm{~g}$ and $\beta \simeq 4.6 \times 10^{-10}$, for $a_{\star}=0$. The left panel shows the evolution of the radiation and PBH energy densities as a function of the SM temperature. PBHs dominate the total energy density between $T=T_{\text {eq }} \simeq 81 \mathrm{GeV}$ and $T=T_{\text {ev }} \simeq 4 \mathrm{MeV}$. Additionally, the right panel shows the oscillation temperature for radiation domination (solid line), and $\mathrm{PBH}$ domination (dotted line). As expected from Eq. (4.6), for a given ALP mass, $T_{\text {osc }}$ decreases for a PBH dominated era. Again, these results were obtained using the numerical code and fit well the analytical estimations.

It is customary to assume that the global PQ symmetry is also anomalous with respect to the electromagnetic gauge group thanks to the existence of exotic vectorlike charged fermions. Consequently, ALPs will couple to two photons, via the effective dimension-five operator

$$
\mathcal{L}_{a \gamma}=-\frac{1}{4} g_{a \gamma} a F_{\mu \nu} \tilde{F}^{\mu \nu}=g_{a \gamma} a \vec{E} \cdot \vec{B},
$$

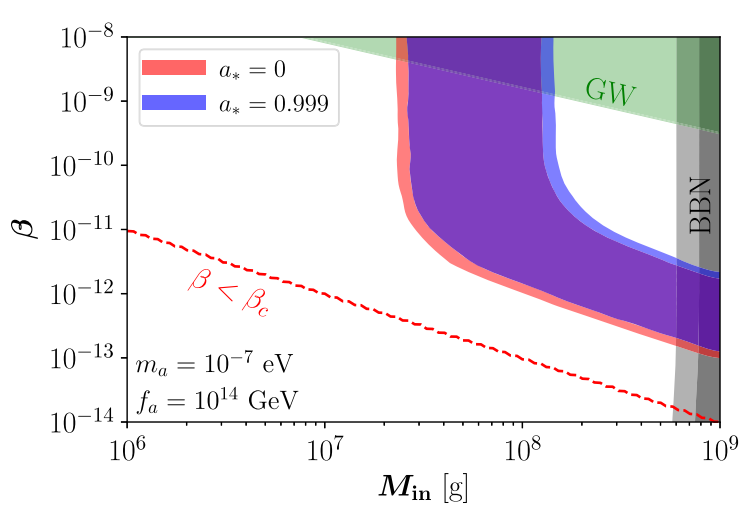

FIG. 1. Parameter space compatible with the whole observed ALP DM abundance, for $m_{a}=10^{-7} \mathrm{eV}$ and $f_{a}=10^{14} \mathrm{GeV}$. The thickness of the bands corresponds to $0.5 \leq \theta_{i} \leq \pi / \sqrt{3}$. The red bands show $a_{\star}=0$, whereas the blue $a_{\star}=0.999$. 



FIG. 2. Left: Evolution of the energy densities for SM radiation and PBHs. Right: Oscillation temperature of ALPs, for radiation domination (solid line) and $\mathrm{PBH}$ domination (dotted line). For both panels, the maximal allowed deviation from the standard cosmological scenario for Schwarzschild PBHs was assumed, i.e., $M_{\text {in }} \simeq 5.7 \times 10^{8} \mathrm{~g}$ and $\beta \simeq 4.6 \times 10^{-10}$.

where the coupling constant $g_{a \gamma}$ is model dependent and related to the breaking scale of the PQ symmetry as

$g_{a \gamma}=\frac{\alpha}{2 \pi f_{a}}\left(\frac{E}{N}-\frac{24+z}{3} \frac{4+z}{1+z} \simeq 10^{-13} \mathrm{GeV}^{-1}\left(\frac{10^{10} \mathrm{GeV}}{f_{a}}\right)\right.$,

with $z \equiv m_{u} / m_{d}$, and $E$ and $N$ are the electromagnetic and color anomalies associated with the ALP anomaly. For Kim-Shifman-Vainshtein-Zakharov (KSVZ) models $E / N=0[83,84]$, whereas for Dine-Fischler-SrednickiZhitnitsky (DFSZ) models $E / N=8 / 3$ [85,86]. The electromagnetic interaction of ALPs is by far the most exploited to look for signatures in observations and experimental searches [87].

Once one assumes that all DM is composed by ALPs, $m_{a}$ and $f_{a}$ (and therefore $g_{a \gamma}$ ) are no longer independent, for a given initial misalignment angle. In Fig. 3 we display the parameter space compatible with the DM relic density constraint, in the plane $\left(m_{a},\left|g_{a \gamma}\right|\right)$. The diagonal band dubbed "ALPs in radiation domination" corresponds to the viable region within the standard cosmology, with the thickness of the band representing the possible values for $\theta_{i}$ in the range $[0.5, \pi / \sqrt{3}]$, with $\theta_{i}=\pi / \sqrt{3}$ being the top border. The regions on the top and bottom give rise to a $\mathrm{DM}$ underabundance and overabundance, respectively. ${ }^{5}$ This parameter space is modified once an early PBH dominated epoch occurs. The red (blue) thick band shows the viable parameter space for $a_{\star}=0 \quad\left(a_{\star}=0.999\right)$, enhanced by the presence of PBHs. The impact of the PBH spin is very mild, and therefore the two bands are almost superimposed. The PBH entropy

\footnotetext{
${ }^{5}$ Notice that we are not considering further production mechanisms of ALPs such as the decay of cosmic strings or domain walls [88-92].
}

injection dilutes the ALP abundance, and therefore higher values for the PQ breaking scale are allowed, which corresponds to lower values for $\left|g_{a \gamma}\right|$. It is interesting to note that for $m_{a} \lesssim 10^{-5} \mathrm{eV}, T_{\text {osc }}<T_{\text {eq }}$, and therefore the ALP relic density is independent on its mass. However, for $m_{a} \gtrsim 10^{-5} \mathrm{eV}, T_{\text {osc }}>T_{\text {eq }}$ and $g_{a \gamma} \propto m_{a}^{1 / 4}$ [cf. Eq. (4.7)].

Figure 3 also overlays in brown current bounds on the ALP-photon coupling. Small masses $\left(m_{a} \lesssim 4 \times 10^{-11} \mathrm{eV}\right)$ are constrained astrophysical black hole spins [94], whereas high masses $\left(m_{a} \gtrsim 10^{2} \mathrm{eV}\right)$ by the x-ray background and extragalactic background light [95]. The masses in the range $10^{-6} \mathrm{eV} \lesssim m_{a} \lesssim 10^{-5} \mathrm{eV}$ are already probed by the so-called haloscope experiments [96], ADMX [97,98], HAYSTAC [99], CAPP [100-102], QUAX [103,104], ORGAN [105], using a highly

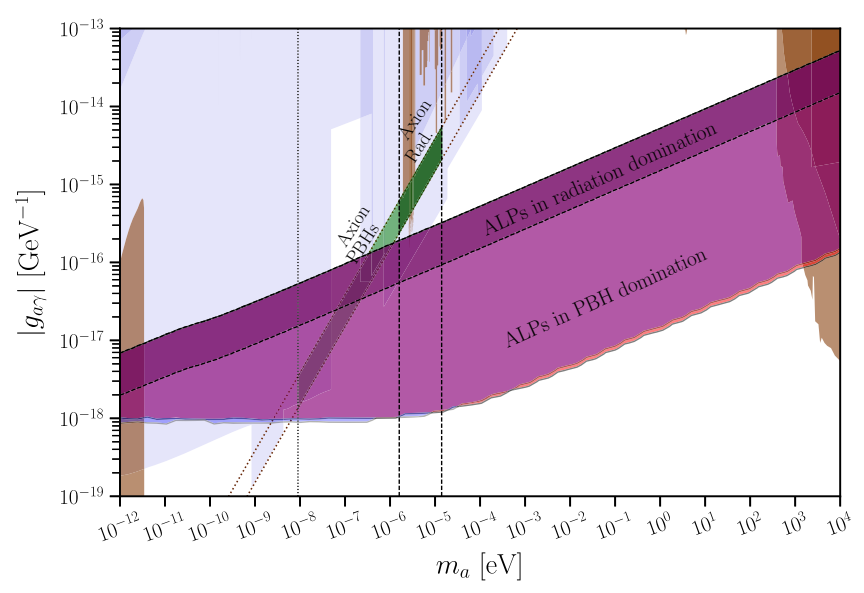

FIG. 3. Viable parameter space for ALPs and the QCD axion, for radiation dominated or PBH dominated scenarios. The brown and light blue shaded regions are the current exclusions and the projected sensitivities of the different experiments described in the text. The figure has been adapted from Ref. [93]. 
tuned microwave cavity that converts ALPs into photons in the presence of a static magnetic field. ${ }^{6}$ Additionally, the light blue regions show projected sensitivities from a number of experiments that count ORGAN [105], MADMAX [106], ALPHA [107], ADMX [108], KLASH [109], DM-Radio [110], and ABRACADABRA $[111,112]$. These bounds have been adapted from Ref. [93]. Even if the PBH domination tends to imply smaller values for $\left|g_{a \gamma}\right|$, this parameter space could be potentially tested in the future by next-generation experiments, especially for light ALP masses, $m_{a} \lesssim 10^{-7} \mathrm{eV}$.

Before closing this section, it is interesting to note that further constraints on $f_{a}$ appear depending on the scale at which the spontaneous breakdown of the PQ symmetry happened. If the PQ symmetry was broken during inflation $\left(f_{a}>H_{I}\right.$, with $H_{I}$ being the inflationary scale) and never restored, the ALP field would be homogenized through the Hubble patch. On the other hand, if the inflationary epoch ends before the PQ transition $\left(f_{a}<H_{I}\right)$ the initial misalignment angle would vary along the different patches of the universe. Let us consider in more detail these two scenarios and their possible relation with PBHs.

\section{A. Preinflationary scenario}

In this case, the PQ symmetry is spontaneously broken during inflation (i.e., $H_{I}<f_{a}$ ), and it is not restored afterwards [32]. The ALP field is homogeneous through various Hubble patches, with a unique value of $\theta_{i}$ characterizing the whole observable universe. In this scenario, the ALP is present during inflation, and therefore ALP isocurvature fluctuations (converted into curvature perturbations) are expected to leave an imprint on the CMB. However, since the CMB measurements do not allow for sizable isocurvature modes, the scale of inflation is pushed to relatively low values. Accordingly, the isocurvature bounds obtained from Planck data [113] impose the lower bound on $f_{a}$ which can be cast as [35]

$$
H_{I} \lesssim 0.9 \times 10^{7} \mathrm{GeV}\left(\frac{\theta_{i}}{\pi}\right)\left(\frac{f_{a}}{10^{11} \mathrm{GeV}}\right)
$$

Since the BBN scale represents a lower bound for $H_{I}$, it follows that $f_{a} \gtrsim 100 \mathrm{GeV} \quad\left(\left|g_{a \gamma}\right| \lesssim 10^{-5} \mathrm{GeV}^{-1}\right)$ for $\theta_{i} \sim \mathcal{O}(1)$, which in turn does not have an impact on the parameter space displayed in Fig. 3. A stronger limit appears when examining the connection with PBHs. Combining the above expression with Eq. (2.1) we obtain a lower limit for the initial mass of the PBH

\footnotetext{
${ }^{6}$ We note that the parameter space shown in Fig. 3 is consistent with an ALP lifetime larger than the age of the universe.
}

$$
\begin{aligned}
M_{\text {in }} & =4 \pi \gamma \frac{M_{P}^{2}}{H\left(T_{\text {in }}\right)} \gtrsim 4 \pi \gamma \frac{M_{P}^{2}}{H_{I}} \\
& \simeq 8.6 \times 10^{6} \mathrm{~g}\left(\frac{1}{\theta_{i}}\right)\left(\frac{10^{11} \mathrm{GeV}}{f_{a}}\right),
\end{aligned}
$$

which reflects the fact that PBHs are created after inflation, in a radiation-dominated epoch. For PBHs fully evaporating before the onset of BBN, $M_{\text {in }} \lesssim 6 \times 10^{8} \mathrm{~g}$, and therefore for $\theta_{i} \sim \mathcal{O}(1)$, it implies that

$$
\left|g_{a \gamma}\right| \lesssim 10^{-12} \mathrm{GeV}^{-1}
$$

which is again automatically satisfied for our relevant parameter space.

\section{B. Postinflationary scenario}

For this case, the PQ symmetry is spontaneously broken after the inflationary period, i.e., $f_{a}<H_{I}$. The misalignment angle takes random values along different Hubble patches, in which case it is averaged out over many patches (in the range $[-\pi, \pi)$ ) so that $\theta_{i}=\sqrt{\left\langle\theta_{i}^{2}\right\rangle}=\pi / \sqrt{3}$. Additionally, the upper limit on the inflationary scale $H_{I}<$ $2.5 \times 10^{-5} M_{P}$ [113] implies that $f_{a} \lesssim 10^{13} \mathrm{GeV}$ (and in turn $g_{a \gamma} \gtrsim 10^{-16} \mathrm{GeV}^{-1}$ ), which finally can be translated into a lower bound on the ALP mass $m_{a} \gtrsim 10^{-7} \mathrm{eV}$, for ALPs being the whole DM.

An important feature of the postinflationary scenario is that the value of the initial misalignment angle changes by a factor of $\mathcal{O}(1)$ from one causal patch to the next. Accordingly, the density of cold ALPs is characterized by sizable inhomogeneities. Their free streaming length is too short to erase these inhomogeneities before the matterradiation equality, so that the density perturbations decouple from the Hubble expansion and start growing by gravitational instability, rapidly forming gravitationally bound objects, called miniclusters [35,58-61].

The formation time of ALP miniclusters is sensitive to the cosmology prior to $\mathrm{BBN}$ and, in particular, to the temperature at which ALP oscillations take place. The scale of ALP minicluster mass is set by the total mass of ALPs within one Hubble volume of radius $R_{\text {osc }} \sim H\left(T_{\text {osc }}\right)^{-1}$, at the time of the matter-radiation equality. Therefore, their mass at formation is

$M_{0}=\frac{4 \pi}{3} \rho_{\mathrm{DM}}\left(T_{0}\right) \frac{s\left(T_{\mathrm{osc}}\right)}{s\left(T_{0}\right)}\left(\frac{1}{H\left(T_{\mathrm{osc}}\right)}\right)^{3} \times \frac{S\left(T_{\mathrm{ev}}\right)}{S\left(T_{\mathrm{osc}}\right)}$.

In a radiation dominated universe and assuming that all relic ALPs bound up in miniclusters, it reduces to

\footnotetext{
${ }^{7}$ When taking into account the anharmonicity of the potential, the average becomes $\theta_{i}=\sqrt{\left\langle\theta_{i}^{2}\right\rangle} \simeq 2.15$ [114].
} 


$$
\begin{aligned}
M_{0} & =\frac{8 g_{\star s}\left(T_{\mathrm{osc}}\right)}{5}\left(\frac{10}{g_{\star}\left(T_{\mathrm{osc}}\right)}\right)^{3 / 2} \frac{\Omega_{\mathrm{DM}} h^{2} \rho_{c} / h^{2}}{s\left(T_{0}\right)} \frac{M_{P}^{3}}{T_{\mathrm{osc}}^{3}} \\
& \simeq 2 \times 10^{-16}\left(\frac{10^{-5} \mathrm{eV}}{m_{a}}\right)^{3 / 2} M_{\odot},
\end{aligned}
$$

as shown in Fig. 4 with a solid black line. The vertical bands are excluded because $f_{a}>H_{I}$, or in tension with the x-ray background and the extragalactic background light [95].

However, if ALPs start to oscillate during the PBH dominated period, the initial minicluster mass can be estimated to be ${ }^{8}$

$$
M_{0} \simeq \frac{16 \pi g_{\star s}}{g_{\star}} \frac{\Omega_{\mathrm{DM}} h^{2} \rho_{c} / h^{2}}{s\left(T_{0}\right)} \times \begin{cases}\pi^{1 / 2}\left(\frac{g_{\star}}{10}\right)^{1 / 4}\left(\frac{M_{P}}{m_{a}}\right)^{3 / 2} \frac{T_{\mathrm{eq}}}{T_{\mathrm{ev}}} & \text { for } T_{\mathrm{osc}} \geq T_{\mathrm{eq}}, \\ \frac{M_{P}^{2}}{m_{a} T_{\mathrm{ev}}} & \text { for } T_{\mathrm{eq}} \geq T_{\mathrm{osc}} \geq T_{\mathrm{ev}}, \\ \pi^{1 / 2}\left(\frac{g_{\star}}{10}\right)^{1 / 4}\left(\frac{M_{P}}{m_{a}}\right)^{3 / 2} & \text { for } T_{\mathrm{ev}} \geq T_{\mathrm{osc}} .\end{cases}
$$

Figure 4 also shows the maximal impact of the $\mathrm{PBH}$ domination era with a broad purple band. Again, we have taken the PBH benchmark values of $M_{\mathrm{in}} \simeq 5.7 \times 10^{8} \mathrm{~g}$ and $\beta \simeq 4.6 \times 10^{-10}$ for Schwarzschild, or $M_{\text {in }} \simeq 7.6 \times 10^{8} \mathrm{~g}$ and $\beta \simeq 3.5 \times 10^{-10}$ for Kerr. The spin of the PBHs has a very limited impact, and therefore the differences in the plot are barely visible. However, it is clear that heavier ALP miniclusters can be formed due to the entropy injection. In other words, the entropy injection works as an enhancement factor [cf. Eq. (4.14)] (see e.g., Refs. [116-118] for related studies). Hence, searches for ALPs, such as indirect detection through gravitational microlensing [119-122], could be extended to regions of the parameter space previously thought to not be relevant.

\section{AXION DARK MATTER}

The production of QCD axion in a cosmology dominated by PBHs was analytically studied in Ref. [45]. For the sake of completeness, here we revisit that scenario from a numerically perspective. In particular, we (i) include the graybody factors in the $\mathrm{PBHs}$ emission properties, (ii) take the effect of the $\mathrm{PBH}$ angular momentum into account, and (iii) numerically solve the evolution of the $\mathrm{PBH}$ and radiation energy density. This is particularly pertinent because it allows one to take into account the timedependent $\mathrm{BH}$ evaporation rate.

For the case of QCD axions, the scale $f_{a}$ at which the PQ $U(1)$ symmetry is spontaneously broken determines the axion mass through the topological susceptibility of QCD [123]

$\chi(T) \simeq 0.0245 \mathrm{fm}^{-4} \times \begin{cases}1 & \text { for } T \leq T_{\mathrm{QCD}} \\ \left(\frac{T}{T_{\mathrm{QCD}}}\right)^{-8.16} & \text { for } T \geq T_{\mathrm{QCD}}\end{cases}$

\footnotetext{
${ }^{8}$ It is worth mentioning that in either a radiation or early matter domination era it may be possible to form ALP miniclusters even in preinflationary scenarios $[115,116]$.
}

as

$$
m_{a}(T)=\frac{\sqrt{\chi(T)}}{f_{a}},
$$

with $T_{\mathrm{QCD}} \simeq 150 \mathrm{MeV}$. As the axion mass is temperature dependent, its abundance in the standard cosmology is

$\Omega_{a} h^{2} \simeq 0.12\left(\frac{\theta_{i}}{10^{-3}}\right)^{2} \times\left\{\begin{array}{l}\left(\frac{m_{a}}{m_{a}^{M^{C D}}}\right)^{-\frac{3}{2}} \text { for } m_{a} \leq m_{a}^{\mathrm{QCD}}, \\ \left(\frac{m_{a}}{m_{a}^{\mathrm{CD}^{\mathrm{CD}}}}\right)^{-\frac{7}{6}} \text { for } m_{a} \geq m_{a}^{\mathrm{QCD}},\end{array}\right.$

with $m_{a}^{\mathrm{QCD}} \equiv m_{a}\left(T_{\mathrm{osc}}=T_{\mathrm{QCD}}\right) \simeq 4.8 \times 10^{-11} \mathrm{eV}$. However, if a PBH-dominated epoch occurs, the axion relic abundance gets modified due to the reduction of its oscillation temperature and its dilution induced by the entropy

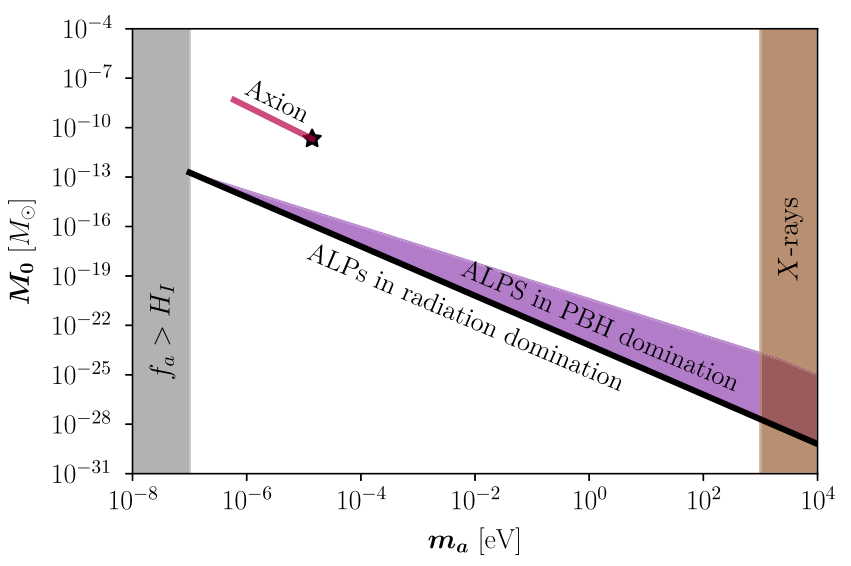

FIG. 4. Minicluster mass in the postinflationary scenario, at the time of formation. ALPs: The thick black line corresponds to the standard cosmological scenario, whereas the purple band to the PBH domination. Axions: The star corresponds to the standard cosmological scenario, whereas the purple line to the $\mathrm{PBH}$ domination. The vertical bands are excluded because $f_{a}>H_{I}$, or in tension with x-ray measurements. 


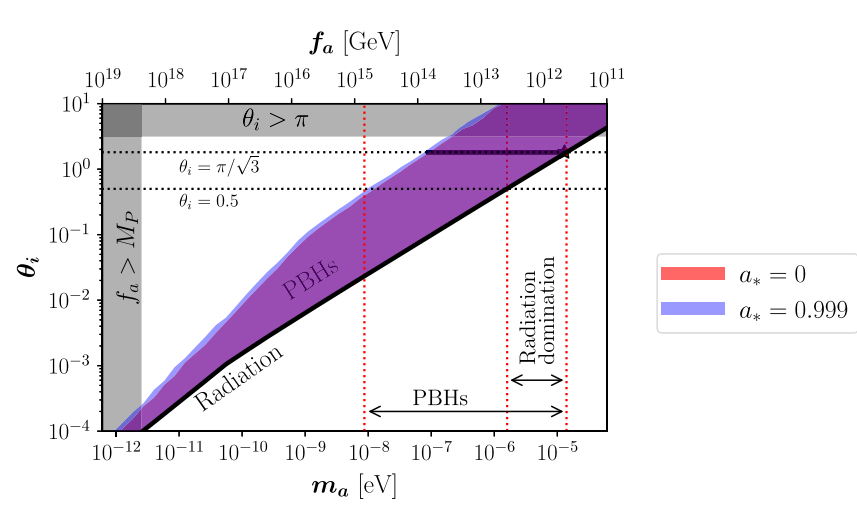

FIG. 5. Initial misalignment angle of QCD axions required to explain the observed DM abundance for $a_{\star}=0$ (red band) and $a_{\star}=0.999$ (blue band). The corresponding values in the standard cosmological scenario are represented by the thick black line.

injection $[45,124,125]$. Again, we numerically solve the coupled Boltzmann equations, determine the dilution factor, and further calculate the modified QCD axion relic density. In Fig. 5, we present our numerical results, which agree well with the analytical estimations presented in Ref. [45]. The purple and blue bands of Fig. 5 show the misalignment angle required to reproduce the whole observed axion DM abundance for the case with Schwarzschild $\left(a_{\star}=0\right)$ and Kerr $\left(a_{\star}=0.999\right)$ PBH domination, respectively. The black thick line corresponds to the standard cosmological scenario. The thickness of the band brackets all possible PBH scenarios compatible with BBN $\left(T_{\mathrm{ev}}>4 \mathrm{MeV}\right)$ and GWs [cf. Eq. (2.6)]. Once we restrict to initial misalignment angles in the range $\theta_{i} \in[0.5, \pi / \sqrt{3}]$, we have that the axion mass can take lower values than those allowed in a purely radiation dominated epoch. Concretely, the axion mass range gets broader toward low values from $\sim\left[10^{-6}, 10^{-5}\right]$ to $\sim\left[10^{-8}, 10^{-5}\right] \mathrm{eV}$. This enlargement of the viable mass range is also shown in Fig. 3, now taking into account the axion coupling to photons. The thicknesses of the band correspond to the KSVZ and DFSZ models for the axion-photon coupling. It is interesting to see that this parameter space is within the reach of future detectors as ABRACADABRA, KLASH, ADMX, and DM-Radio.

\section{A. Preinflationary scenario}

When the axion is present during inflation (and the PQ symmetry is not restored after it), the requirement of axions to constitute all DM along with the standard cosmological scenario along with the isocurvature bound (4.11) leads to

$$
H_{I} \lesssim \begin{cases}3.2 \times 10^{9} \mathrm{GeV}\left(\frac{f_{a}}{10^{17} \mathrm{GeV}}\right)^{1 / 4} & \text { for } f_{a} \gtrsim 1.2 \times 10^{17} \mathrm{GeV} \\ 2.6 \times 10^{7} \mathrm{GeV}\left(\frac{f_{a}}{10^{12} \mathrm{GeV}}\right)^{5 / 12} & \text { for } f_{a} \lesssim 1.2 \times 10^{17} \mathrm{GeV}\end{cases}
$$

Consequently, under the PBH domination slightly higher inflation scales can be reached since $f_{a}$ is allowed to take higher values. In other words, the relevant parameter space displayed in Figs. 3 and 5 does not get affected. Translating the above bounds into the initial mass for the PBHs, we have

$$
M_{\text {in }} \gtrsim \begin{cases}7.5 \times 10^{3} \mathrm{~g}\left(\frac{10^{17} \mathrm{GeV}}{f_{a}}\right)^{1 / 4} & \text { for } f_{a} \gtrsim 1.2 \times 10^{17} \mathrm{GeV}, \\ 9.4 \times 10^{5} \mathrm{~g}\left(\frac{10^{12} \mathrm{GeV}}{f_{a}}\right)^{5 / 12} & \text { for } f_{a} \lesssim 1.2 \times 10^{17} \mathrm{GeV} .\end{cases}
$$

Contrary to the ALP case, there is no significant lower bound on $f_{a}$ when $M_{\text {in }}$ approaches the maximum value allowed by BBN.

\section{B. Postinflationary scenario}

Since the PQ symmetry remains conserved during the inflation period $\left(f_{a}<H_{I}\right)$, the axion field does not get homogenized. The upper limit on the inflationary scale implies that $f_{a} \lesssim 10^{13} \mathrm{GeV}$, and hence the lower bound on the axion mass $m_{a} \gtrsim 6 \times 10^{-7} \mathrm{eV}$.

In the standard cosmological scenario, axions start oscillating at the temperature $T_{\text {osc }} \simeq 1.1 \mathrm{GeV}$. Therefore, if the misalignment mechanism is the unique method to produce axions, they should have a mass $m_{a} \simeq 1.8 \times$ $10^{-5} \mathrm{eV}$ (for $\theta_{i}=\pi / \sqrt{3}$ ) in order to account for the whole of the DM abundance. This case is identified by a star in the upper right region in Fig. 5. This result is remarkably modified by the dilution factor $S\left(T_{\mathrm{osc}}\right) / S\left(T_{\mathrm{ev}}\right)$ that emerges within the PBH domination, which now allows for a mass range instead of a single point, reaching values up to 2 orders of magnitude lower, i.e., $\mathcal{O}\left(10^{-7}\right) \mathrm{eV}$. This new range is represented by the thick solid horizon line in Fig. 5. 
The gravitational clump of axion density inhomogeneities at the time of radiation-matter equality leads to miniclusters having a mass $M_{0} \simeq 2.1 \times 10^{-11} M_{\odot}$ in the standard cosmological scenario (see the star mark in
Fig. 4). However, in a PBH dominated scenario, such a mass gets enhanced due to the entropy injection by $\mathrm{BH}$ evaporation. The mass $M_{0}$ of the axion miniclusters at formation becomes

$$
M_{0} \simeq \frac{16 g_{\star s}}{g_{\star}} \frac{\Omega h^{2} \rho_{c} / h^{2}}{s\left(T_{0}\right)} \times \begin{cases}\pi^{1 / 2}\left(\frac{10}{g_{\star}}\right)^{1 / 4} \frac{M_{P}^{5 / 2}}{T_{\mathrm{QCD}}^{2} m_{a}^{1 / 2}} \frac{T_{\mathrm{eq}}}{T_{\mathrm{ev}}} & \text { for } T_{\mathrm{osc}} \geq T_{\mathrm{eq}}, \\ \pi^{3 / 11}\left(\frac{10}{g_{\star}}\right)^{4 / 11} \frac{M_{P}^{30 / 11}}{T_{\mathrm{QCD}}^{12 / 1} m_{a}^{3 / 11} T_{\mathrm{eq}}^{4 / 11} T_{\mathrm{ev}}} & \text { for } T_{\mathrm{eq}} \geq T_{\mathrm{osc}} \geq T_{\mathrm{c}} \\ \pi^{1 / 2}\left(\frac{10}{g_{\star}}\right)^{1 / 4} \frac{M_{P}^{5 / 2}}{T_{\mathrm{QCD}}^{2} m_{a}^{1 / 2}} & \text { for } T_{\mathrm{c}} \geq T_{\mathrm{osc}}\end{cases}
$$

In Fig. 4 we show the enhancement on $M_{0}$ (purple line) generated by the $\mathrm{PBH}$ domination. It is remarkable that now $M_{0}$ can reach values as high as $10^{-8} M_{\odot}$.

\section{DARK RADIATION}

Axions and ALPs emitted from PBH evaporation are ultrarelativistic, thus behaving as DR and contributing to the effective number of neutrinos $[5,23,35,44]$. We note that ALP DR directly emitted from nonrotating PBHs was analyzed in Ref. [44] recently. Here we revisit ALP DR from $\mathrm{PBHs}$ with a more general perspective; namely we will study both nonrotating and rotating PBHs. The contribution to the effective number of neutrinos can be simply estimated, obtaining

$$
\begin{aligned}
\Delta N_{\text {eff }}= & {\left[\frac{8}{7}\left(\frac{11}{4}\right)^{\frac{4}{3}}+N_{\text {eff }}^{\mathrm{SM}}\right] \frac{\rho_{a}\left(T_{\mathrm{ev}}\right)}{\rho_{R}\left(T_{\mathrm{ev}}\right)}\left(\frac{g_{\star}\left(T_{\mathrm{ev}}\right)}{g_{\star}\left(T_{\text {equality }}\right)}\right) } \\
& \times\left(\frac{g_{\star s}\left(T_{\text {equality }}\right)}{g_{\star s}\left(T_{\text {ev }}\right)}\right)^{\frac{4}{3}},
\end{aligned}
$$

where $T_{\text {equality }} \simeq 0.75 \mathrm{eV}$ denotes the temperature at (late) matter-radiation equality. In the PBH-dominated scenario, the ratio between the axion (or ALP) and the radiation energy densities is related to their contributions to the evaporation function

$$
\frac{\rho_{a}\left(T_{\mathrm{ev}}\right)}{\rho_{R}\left(T_{\mathrm{ev}}\right)}=\frac{\varepsilon_{a}\left(z_{i}, a_{\star}\right)}{\varepsilon_{R}\left(z_{i}, a_{\star}\right)},
$$

with $\varepsilon_{a}\left(T_{\mathrm{ev}}\right)$ being the axion evaporation function, and $\varepsilon_{R}\left(T_{\mathrm{ev}}\right)$ the total SM radiation contribution to the evaporation function. For the case of Schwarzschild PBHs, $\Delta N_{\text {eff }}$ is simply

$$
\Delta N_{\mathrm{eff}} \simeq 0.237 \frac{g_{\star}\left(T_{\mathrm{ev}}\right)}{g_{\star s}\left(T_{\mathrm{ev}}\right)^{\frac{4}{3}}},
$$

which is independent from $\beta$, and consistent with the result obtained in Ref. [44]. For Kerr PBHs, the time depletion of the angular momentum makes it more difficult to obtain a simple analytical form.

Left (right) panel of Fig. 6 shows the contribution to $\Delta N_{\text {eff }}$ as a function of $\mathrm{BH}$ mass and $\beta$ for Schwarzschild (Kerr) PBHs. Let us note that in a PBH-dominated era, $\Delta N_{\text {eff }}$ tends to be independent of $\beta$, as noted before [cf. Eq. (6.3)]. On the contrary, when $\beta<\beta_{c}$, larger $M_{\text {in }}$ is needed with smaller $\beta$ in order to yield a fixed $\Delta N_{\text {eff }}$. We also note that when $M_{\text {in }} \simeq 2 \times 10^{7} \mathrm{~g}$ (correspondingly $T_{\mathrm{ev}} \simeq 0.1 \mathrm{GeV}$ ), there are some features in the lines shown in Fig. 6. This is because of the sharp change of $g_{\star}$ and $g_{\star s}$ due to QCD phase transition [5,23]. Comparing the resulting values of $\Delta N_{\text {eff }}$ for Schwarzschild and Kerr $\mathrm{PBH}$, we observe that for the latter case the final values are smaller than those for nonrotating PBHs. This is simply related to the properties of Kerr PBHs: if the $\mathrm{BH}$ spin is close to maximal, the emission of higher spin particles is enhanced in the early stages of the evaporation [71]. Thus, the particle injection to SM radiation density is larger than in the Schwarzschild case. Meanwhile, the axion/ALP emission is not affected by the BH spin, so their final contribution to $\Delta N_{\text {eff }}$ is in fact reduced $[12,126]$. The current upper bound on $\Delta N_{\text {eff }}$ comes from the Planck Collaboration, and reads $N_{\text {eff }}=2.99 \pm 0.17$ [76]. However, $\Delta N_{\text {eff }}$ due to emitted hot axions/ALPs could reach the sensitivity of next generation CMB experiments: CMB-S4 [63] ( $\left.\Delta N_{\text {eff }} \sim 0.06\right)$, PICO [64] $\left(\Delta N_{\text {eff }} \sim 0.06\right)$, and SPT-3G/SO [65,66] $\left(\Delta N_{\text {eff }} \sim 0.1\right)$, and therefore axion/ALP DR could be tested in the near future. ${ }^{9}$

\footnotetext{
${ }^{9}$ It is interesting to note that extra radiation, at the level of $0.2 \lesssim \Delta N_{\text {eff }} \lesssim 0.5$, can alleviate the tension between measurements of the Hubble parameter at early and late times [5,76,127-130]. However, such large levels cannot be reached by PBHs.
} 

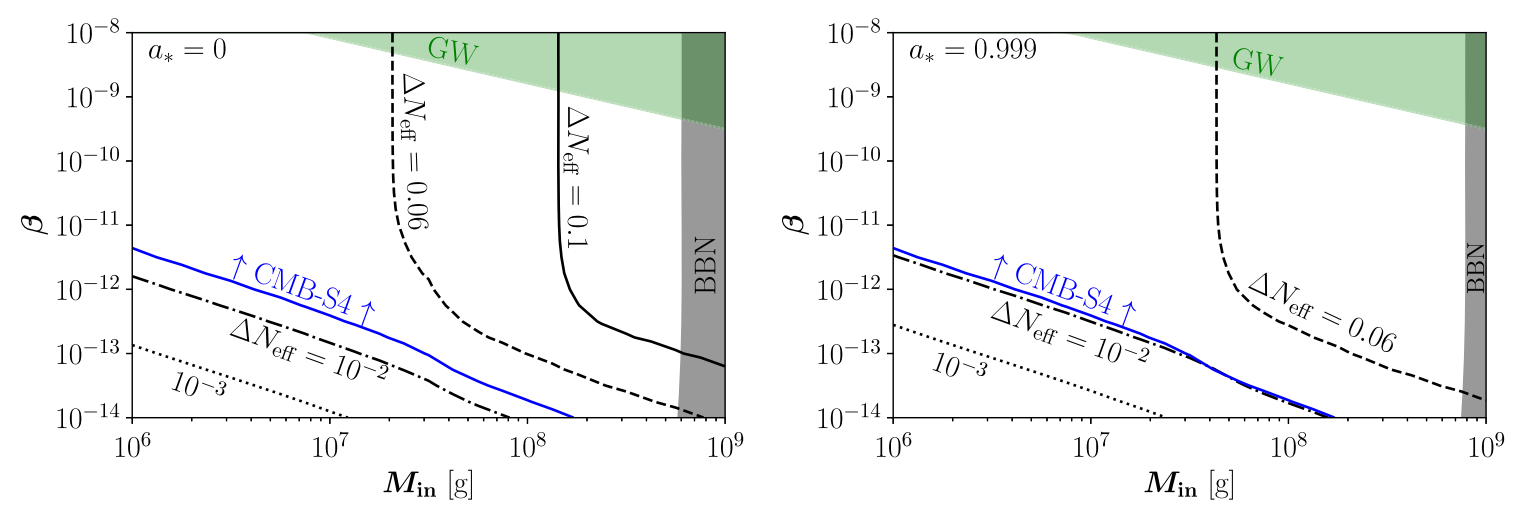

FIG. 6. Contribution to the effective number of neutrinos $\Delta N_{\text {eff }}$ coming from axions/ALPs directly emitted via Hawking radiation for $a_{\star}=0$ (left) and $a_{\star}=0.999$ (right). The blue line indicates the region within the reach of the future CMB-S4 experiment [63].

\section{CONCLUSIONS}

PBHs are without doubt one of the most interesting objects that could exist in nature. As the PBH energy density scale as nonrelativistic matter, they can naturally dominate the expansion rate of the Universe, triggering a nonstandard cosmological epoch. Moreover, they also source all types of particles, thus injecting large quantities of entropy to the primordial plasma. Hence, these two specific properties, namely, the possibility of the dominating expansion of the universe and the large injection of entropy, make the phenomenology of a PBH dominated universe unique among the possible scenarios for a nonstandard cosmology.

In this paper, we studied the phenomenological consequences of ALPs as dark matter candidates in an early PBH-dominated epoch. To that end, we numerically solved the set of Boltzmann equations for the background dynamics, carefully taking into account both the graybody factors and the $\mathrm{PBH}$ angular momentum. PBHs have a strong impact on the misalignment ALP production. On the one hand, as the Hubble expansion rate is enhanced, the oscillation temperature decreases, which corresponds to a delay in the oscillations. On the other hand, the entropy injection due to the PBH evaporation has to be compensated by a larger spontaneous breaking scale value of the Peccei-Quinn symmetry or, equivalently, by a smaller ALPphoton coupling. An equivalent trend happens for QCD axions, with the particularity that its standard mass window $m_{a} \simeq\left[10^{-6}-10^{-5}\right] \mathrm{eV}$ gets broader to $\sim\left[10^{-8}-10^{-5}\right] \mathrm{eV}$ [for misalignment angles of order $\mathcal{O}(1)$ ], mainly due to the entropy injection. It is interesting to note that for both ALPs and axions, the new viable parameter space is within the projected sensitivities of detectors as ABRACADABRA, KLASH, ADMX, and DM-Radio.

Additionally, we showed that at production the ALP/ axion minicluster mass could increase by several orders of magnitude, due to the enhancement required by the $\mathrm{PBH}$ entropy injection. This could give new prospects for the indirect detection via gravitational microlensing of ALP and axion dark matter, with respect to the standard cosmological scenario.

Finally, we analyzed in detail the dark radiation arising from relativistic axions and ALPs directly emitted from PBHs evaporation. For Kerr PBH, the contributions $\Delta N_{\text {eff }}$ are smaller than those for Schwarzschild PBHs. Interestingly, this contribution is within the projected reach of future CMB Stage 4 experiments and could help to relax the tension between late and early-time Hubble determinations.

\section{ACKNOWLEDGMENTS}

N.B. received funding from the Spanish FEDER/ MCIU-AEI under Grant No. FPA2017-84543-P, and the Patrimonio Autónomo-Fondo Nacional de Financiamiento para la Ciencia, la Tecnología y la Innovación Francisco José de Caldas (MinCienciasColombia) Grant No. 80740-465-2020. The work of O. Z. is supported by Sostenibilidad-UdeA and the UdeA/CODI Grants No. 2017-16286 and No. 2020-3317. This project has received funding/support from the European Union's Horizon 2020 research and innovation programme under the Marie Skłodowska-Curie Grant Agreement No. 860881HIDDeN. Fermilab is operated by the Fermi Research Alliance, LLC under Contract No. DE-AC02-07CH11359 with the United States Department of Energy. 
[1] B. Carr, K. Kohri, Y. Sendouda, and J. Yokoyama, Constraints on primordial black holes, arXiv:2002.12778.

[2] B. Carr and F. Kuhnel, Primordial black holes as dark matter: Recent developments, Annu. Rev. Nucl. Part. Sci. 70, 355 (2020).

[3] P. Villanueva-Domingo, O. Mena, and S. Palomares-Ruiz, A brief review on primordial black holes as dark matter, Front. Astron. Space Sci. 8, 87 (2021).

[4] B. Carr and F. Kuhnel, Primordial black holes as dark matter candidates, arXiv:2110.02821.

[5] D. Hooper, G. Krnjaic, and S. D. McDermott, Dark radiation and superheavy dark matter from black hole domination, J. High Energy Phys. 08 (2019) 001.

[6] I. Masina, Dark matter and dark radiation from evaporating primordial black holes, Eur. Phys. J. Plus 135, 552 (2020).

[7] I. Baldes, Q. Decant, D. C. Hooper, and L. Lopez-Honorez, Non-cold dark matter from primordial black hole evaporation, J. Cosmol. Astropart. Phys. 08 (2020) 045.

[8] P. Gondolo, P. Sandick, and B. Shams Es Haghi, Effects of primordial black holes on dark matter models, Phys. Rev. D 102, 095018 (2020).

[9] N. Bernal and Ó. Zapata, Self-interacting dark matter from primordial black holes, J. Cosmol. Astropart. Phys. 03 (2021) 007.

[10] N. Bernal and Ó. Zapata, Gravitational dark matter production: primordial black holes and UV freeze-in, Phys. Lett. B 815, 136129 (2021).

[11] N. Bernal and Ó. Zapata, Dark matter in the time of primordial black holes, J. Cosmol. Astropart. Phys. 03 (2021) 015.

[12] I. Masina, Dark matter and dark radiation from evaporating Kerr primordial black holes, arXiv:2103.13825.

[13] A. Arbey, J. Auffinger, P. Sandick, B. Shams Es Haghi, and K. Sinha, Precision calculation of dark radiation from spinning primordial black holes and early matterdominated eras, Phys. Rev. D 103, 123549 (2021).

[14] S. Jyoti Das, D. Mahanta, and D. Borah, Low scale leptogenesis and dark matter in the presence of primordial black holes, J. Cosmol. Astropart. Phys. 11 (2021) 019.

[15] A. Cheek, L. Heurtier, Y. F. Perez-Gonzalez, and J. Turner, Primordial black hole evaporation and dark matter production: I. Solely hawking radiation, arXiv:2107.00013.

[16] A. Cheek, L. Heurtier, Y. F. Perez-Gonzalez, and J. Turner, Primordial black hole evaporation and dark matter production: II. Interplay with the freeze-in/out mechanism, arXiv:2107.00016.

[17] P. Sandick, B. S. Es Haghi, and K. Sinha, Asymmetric reheating by primordial black holes, Phys. Rev. D 104, 083523 (2021).

[18] J. D. Barrow, E. J. Copeland, E. W. Kolb, and A. R. Liddle, Baryogenesis in extended inflation. 2. Baryogenesis via primordial black holes, Phys. Rev. D 43, 984 (1991).

[19] Y. Hamada and S. Iso, Baryon asymmetry from primordial black holes, Prog. Theor. Exp. Phys. 2017, 033B02 (2017).

[20] D. Hooper and G. Krnjaic, GUT baryogenesis with primordial black holes, Phys. Rev. D 103, 043504 (2021).

[21] Y. F. Perez-Gonzalez and J. Turner, Assessing the tension between a black hole dominated early Universe and leptogenesis, Phys. Rev. D 104, 103021 (2021).
[22] S. Datta, A. Ghosal, and R. Samanta, Baryogenesis from ultralight primordial black holes and strong gravitational waves from cosmic strings, J. Cosmol. Astropart. Phys. 08 (2021) 021.

[23] C. Lunardini and Y. F. Perez-Gonzalez, Dirac and Majorana neutrino signatures of primordial black holes, J. Cosmol. Astropart. Phys. 08 (2020) 014.

[24] P. Auclair and V. Vennin, Primordial black holes from metric preheating: mass fraction in the excursion-set approach, J. Cosmol. Astropart. Phys. 02 (2021) 038.

[25] S. Weinberg, A New Light Boson?, Phys. Rev. Lett. 40, 223 (1978).

[26] F. Wilczek, Problem of Strong $P$ and $T$ Invariance in the Presence of Instantons, Phys. Rev. Lett. 40, 279 (1978).

[27] R. D. Peccei and H. R. Quinn, CP Conservation in the Presence of Instantons, Phys. Rev. Lett. 38, 1440 (1977).

[28] R. D. Peccei and H. R. Quinn, Some aspects of instantons, Nuovo Cimento A 41, 309 (1977).

[29] R. D. Peccei and H. R. Quinn, Constraints imposed by $C P$ conservation in the presence of instantons, Phys. Rev. D 16, 1791 (1977).

[30] J. Preskill, M. B. Wise, and F. Wilczek, Cosmology of the invisible axion, Phys. Lett. 120B, 127 (1983).

[31] L. F. Abbott and P. Sikivie, A cosmological bound on the invisible axion, Phys. Lett. B 120B, 133 (1983).

[32] M. Dine and W. Fischler, The not so harmless axion, Phys. Lett. 120B, 137 (1983).

[33] P. Sikivie, Axion cosmology, Lect. Notes Phys. 741, 19 (2008).

[34] D. J. E. Marsh, Axion cosmology, Phys. Rep. 643, 1 (2016).

[35] L. Di Luzio, M. Giannotti, E. Nardi, and L. Visinelli, The landscape of QCD axion models, Phys. Rep. 870, 1 (2020).

[36] P. Sikivie, Invisible axion search methods, Rev. Mod. Phys. 93, 015004 (2021).

[37] F. Takahashi, W. Yin, and A. H. Guth, QCD axion window and low-scale inflation, Phys. Rev. D 98, 015042 (2018).

[38] S.-Y. Ho, F. Takahashi, and W. Yin, Relaxing the cosmological moduli problem by low-scale inflation, J. High Energy Phys. 04 (2019) 149.

[39] S. Bird, I. Cholis, J. B. Muñoz, Y. Ali-Haïmoud, M. Kamionkowski, E. D. Kovetz, A. Raccanelli, and A. G. Riess, Did LIGO Detect Dark Matter?, Phys. Rev. Lett. 116, 201301 (2016).

[40] M. Sasaki, T. Suyama, T. Tanaka, and S. Yokoyama, Primordial Black Hole Scenario for the GravitationalWave Event GW150914, Phys. Rev. Lett. 117, 061101 (2016).

[41] S. Clesse and J. García-Bellido, The clustering of massive primordial black holes as dark matter: Measuring their mass distribution with Advanced LIGO, Phys. Dark Universe 15, 142 (2017).

[42] S. Clesse and J. García-Bellido, Seven hints for primordial black hole dark matter, Phys. Dark Universe 22, 137 (2018).

[43] A. D. Gow, C. T. Byrnes, A. Hall, and J. A. Peacock, Primordial black hole merger rates: Distributions for multiple LIGO observables, J. Cosmol. Astropart. Phys. 01 (2020) 031. 
[44] F. Schiavone, D. Montanino, A. Mirizzi, and F. Capozzi, Axion-like particles from primordial black holes shining through the Universe, J. Cosmol. Astropart. Phys. 08 (2021) 063.

[45] N. Bernal, F. Hajkarim, and Y. Xu, Axion dark matter in the time of primordial black holes, Phys. Rev. D 104, 075007 (2021).

[46] J. Jaeckel and A. Ringwald, The low-energy frontier of particle physics, Annu. Rev. Nucl. Part. Sci. 60, 405 (2010).

[47] P. Arias, D. Cadamuro, M. Goodsell, J. Jaeckel, J. Redondo, and A. Ringwald, WISPy cold dark matter, J. Cosmol. Astropart. Phys. 06 (2012) 013.

[48] A. Ringwald, Exploring the role of axions and other WISPs in the dark Universe, Phys. Dark Universe 1, 116 (2012).

[49] I. G. Irastorza and J. Redondo, New experimental approaches in the search for axion-like particles, Prog. Part. Nucl. Phys. 102, 89 (2018).

[50] P. Svrcek and E. Witten, Axions in string theory, J. High Energy Phys. 06 (2006) 051.

[51] A. Arvanitaki, S. Dimopoulos, S. Dubovsky, N. Kaloper, and J. March-Russell, String axiverse, Phys. Rev. D 81, 123530 (2010).

[52] N. Blinov, M. J. Dolan, P. Draper, and J. Kozaczuk, Dark matter targets for axionlike particle searches, Phys. Rev. D 100, 015049 (2019).

[53] K. Inomata, M. Kawasaki, K. Mukaida, T. Terada, and T. T. Yanagida, Gravitational wave production right after a primordial black hole evaporation, Phys. Rev. D 101, 123533 (2020).

[54] T. Papanikolaou, V. Vennin, and D. Langlois, Gravitational waves from a universe filled with primordial black holes, J. Cosmol. Astropart. Phys. 03 (2021) 053.

[55] G. Domènech, C. Lin, and M. Sasaki, Gravitational wave constraints on the primordial black hole dominated early universe, J. Cosmol. Astropart. Phys. 04 (2021) 062.

[56] G. Domènech, V. Takhistov, and M. Sasaki, Exploring evaporating primordial black holes with gravitational waves, Phys. Lett. B 823, 136722 (2021).

[57] G. Domènech, Scalar induced gravitational waves review, Universe 7, 398 (2021).

[58] C. J. Hogan and M. J. Rees, Axion miniclusters, Phys. Lett. B 205, 228 (1988).

[59] E. W. Kolb and I. I. Tkachev, Nonlinear axion dynamics and formation of cosmological pseudosolitons, Phys. Rev. D 49, 5040 (1994).

[60] E. W. Kolb and I. I. Tkachev, Axion Miniclusters and Bose Stars, Phys. Rev. Lett. 71, 3051 (1993).

[61] E. W. Kolb and I. I. Tkachev, Large amplitude isothermal fluctuations and high density dark matter clumps, Phys. Rev. D 50, 769 (1994).

[62] B. Eggemeier, J. Redondo, K. Dolag, J. C. Niemeyer, and A. Vaquero, First Simulations of Axion Minicluster Halos, Phys. Rev. Lett. 125, 041301 (2020).

[63] CMB-S4 Collaboration, CMB-S4 science book, first edition, arXiv: 1610.02743.

[64] NASA PICO Collaboration, PICO: Probe of inflation and cosmic origins, arXiv:1902.10541.
[65] SPT-3G Collaboration, SPT-3G: A next-generation cosmic microwave background polarization experiment on the south pole telescope, Proc. SPIE Int. Soc. Opt. Eng. 9153, 91531P (2014).

[66] Simons Observatory Collaboration, The simons observatory: Science goals and forecasts, J. Cosmol. Astropart. Phys. 02 (2019) 056.

[67] S. W. Hawking, Particle creation by black holes, Commun. Math. Phys. 43, 199 (1975).

[68] B. J. Carr and S. W. Hawking, Black holes in the early Universe, Mon. Not. R. Astron. Soc. 168, 399 (1974).

[69] W. H. Press and P. Schechter, Formation of galaxies and clusters of galaxies by selfsimilar gravitational condensation, Astrophys. J. 187, 425 (1974).

[70] B. J. Carr, K. Kohri, Y. Sendouda, and J. Yokoyama, New cosmological constraints on primordial black holes, Phys. Rev. D 81, 104019 (2010).

[71] D. N. Page, Particle emission rates from a black hole: Massless particles from an uncharged, nonrotating hole, Phys. Rev. D 13, 198 (1976).

[72] J. H. MacGibbon and B. R. Webber, Quark and gluon jet emission from primordial black holes: The instantaneous spectra, Phys. Rev. D 41, 3052 (1990).

[73] J. H. MacGibbon, Quark and gluon jet emission from primordial black holes. 2. The lifetime emission, Phys. Rev. D 44, 376 (1991).

[74] R. Allahverdi et al., The first three seconds: A review of possible expansion histories of the early Universe, Open J. Astrophys. 4, 1 (2020).

[75] F. W. Stecker and Q. Shafi, The Evolution of Structure in the Universe From Axions, Phys. Rev. Lett. 50, 928 (1983).

[76] Planck Collaboration, Planck 2018 results. VI. Cosmological parameters, Astron. Astrophys. 641, A6 (2020).

[77] S. Sarkar, Big bang nucleosynthesis and physics beyond the standard model, Rep. Prog. Phys. 59, 1493 (1996).

[78] M. Kawasaki, K. Kohri, and N. Sugiyama, Cosmological Constraints on Late Time Entropy Production, Phys. Rev. Lett. 82, 4168 (1999).

[79] M. Kawasaki, K. Kohri, and N. Sugiyama, MeV scale reheating temperature and thermalization of neutrino background, Phys. Rev. D 62, 023506 (2000).

[80] S. Hannestad, What is the lowest possible reheating temperature?, Phys. Rev. D 70, 043506 (2004).

[81] P. F. de Salas, M. Lattanzi, G. Mangano, G. Miele, S. Pastor, and O. Pisanti, Bounds on very low reheating scenarios after Planck, Phys. Rev. D 92, 123534 (2015).

[82] T. Hasegawa, N. Hiroshima, K. Kohri, R. S. L. Hansen, T. Tram, and S. Hannestad, $\mathrm{MeV}$-scale reheating temperature and thermalization of oscillating neutrinos by radiative and hadronic decays of massive particles, J. Cosmol. Astropart. Phys. 12 (2019) 012.

[83] J.E. Kim, Weak Interaction Singlet and Strong $C P$ Invariance, Phys. Rev. Lett. 43, 103 (1979).

[84] M. A. Shifman, A. I. Vainshtein, and V. I. Zakharov, Can confinement ensure natural $C P$ invariance of strong interactions?, Nucl. Phys. B166, 493 (1980).

[85] A. R. Zhitnitsky, On possible suppression of the axion hadron interactions. (In Russian), Sov. J. Nucl. Phys. 31, 260 (1980). 
[86] M. Dine, W. Fischler, and M. Srednicki, A simple solution to the strong $C P$ problem with a harmless axion, Phys. Lett. 104B, 199 (1981).

[87] P. W. Graham, I. G. Irastorza, S. K. Lamoreaux, A. Lindner, and K. A. van Bibber, Experimental searches for the axion and axion-like particles, Annu. Rev. Nucl. Part. Sci. 65, 485 (2015).

[88] R. L. Davis, Goldstone bosons in string models of galaxy formation, Phys. Rev. D 32, 3172 (1985).

[89] D. Harari and P. Sikivie, On the evolution of global strings in the early Universe, Phys. Lett. B 195, 361 (1987).

[90] R. A. Battye and E. P. S. Shellard, Axion String Constraints, Phys. Rev. Lett. 73, 2954 (1994).

[91] T. Hiramatsu, M. Kawasaki, K. Saikawa, and T. Sekiguchi, Production of dark matter axions from collapse of stringwall systems, Phys. Rev. D 85, 105020 (2012).

[92] G. B. Gelmini, A. Simpson, and E. Vitagliano, Gravitational waves from axionlike particle cosmic string-wall networks, Phys. Rev. D 104, L061301 (2021).

[93] C. O'Hare, cajohare/axionlimits: Axionlimits, (2020), 10.5281/zenodo.3932430.

[94] V. M. Mehta, M. Demirtas, C. Long, D. J. E. Marsh, L. Mcallister, and M. J. Stott, Superradiance exclusions in the landscape of type IIB string theory, arXiv:2011.08693.

[95] D. Cadamuro and J. Redondo, Cosmological bounds on pseudo Nambu-Goldstone bosons, J. Cosmol. Astropart. Phys. 02 (2012) 032.

[96] P. Sikivie, Experimental Tests of the Invisible Axion, Phys. Rev. Lett. 51, 1415 (1983).

[97] ADMX Collaboration, A SQUID-Based Microwave Cavity Search for Dark-Matter Axions, Phys. Rev. Lett. 104, 041301 (2010).

[98] ADMX Collaboration, Extended Search for the Invisible Axion with the Axion Dark Matter Experiment, Phys. Rev. Lett. 124, 101303 (2020).

[99] HAYSTAC Collaboration, Results from phase 1 of the HAYSTAC microwave cavity axion experiment, Phys. Rev. D 97, 092001 (2018).

[100] S. Lee, S. Ahn, J. Choi, B. R. Ko, and Y. K. Semertzidis, Axion Dark Matter Search Around 6.7 $\mu \mathrm{eV}$, Phys. Rev. Lett. 124, 101802 (2020).

[101] J. Jeong, S. Youn, S. Bae, J. Kim, T. Seong, J. E. Kim, and Y. K. Semertzidis, Search for Invisible Axion Dark Matter with a Multiple-Cell Haloscope, Phys. Rev. Lett. 125, 221302 (2020).

[102] CAPP Collaboration, First Results from an Axion Haloscope at CAPP around $10.7 \mu \mathrm{eV}$, Phys. Rev. Lett. 126, 191802 (2021).

[103] D. Alesini et al., Galactic axions search with a superconducting resonant cavity, Phys. Rev. D 99, 101101 (2019).

[104] D. Alesini et al., Search for invisible axion dark matter of mass $m_{a}=43 \mu \mathrm{eV}$ with the QUAX-ar experiment, Phys. Rev. D 103, 102004 (2021).

[105] B. T. McAllister, G. Flower, E. N. Ivanov, M. Goryachev, J. Bourhill, and M. E. Tobar, The ORGAN experiment: An axion haloscope above $15 \mathrm{GHz}$, Phys. Dark Universe 18, 67 (2017).

[106] S. Beurthey et al., MADMAX status report, arXiv: 2003.10894.
[107] M. Lawson, A. J. Millar, M. Pancaldi, E. Vitagliano, and F. Wilczek, Tunable Axion Plasma Haloscopes, Phys. Rev. Lett. 123, 141802 (2019).

[108] I. Stern, ADMX status, Proc. Sci. ICHEP2016 (2016) 198 [arXiv:1612.08296].

[109] D. Alesini, D. Babusci, D. Di Gioacchino, C. Gatti, G. Lamanna, and C. Ligi, The KLASH proposal, arXiv:1707.06010.

[110] S. Chaudhuri, K. Irwin, P. W. Graham, and J. Mardon, Optimal impedance matching and quantum limits of electromagnetic axion and hidden-photon dark matter searches, arXiv:1803.01627.

[111] Y. Kahn, B. R. Safdi, and J. Thaler, Broadband and Resonant Approaches to Axion Dark Matter Detection, Phys. Rev. Lett. 117, 141801 (2016).

[112] J. L. Ouellet et al., First Results from ABRACADABRA$10 \mathrm{~cm}$ : A Search for Sub- $\mu \mathrm{eV}$ Axion Dark Matter, Phys. Rev. Lett. 122, 121802 (2019).

[113] Planck Collaboration, Planck 2018 results. X. Constraints on inflation, Astron. Astrophys. 641, A10 (2020).

[114] G. Grilli di Cortona, E. Hardy, J. Pardo Vega, and G. Villadoro, The QCD axion, precisely, J. High Energy Phys. 01 (2016) 034.

[115] E. Hardy, Miniclusters in the axiverse, J. High Energy Phys. 02 (2017) 046.

[116] A. E. Nelson and H. Xiao, Axion cosmology with early matter domination, Phys. Rev. D 98, 063516 (2018).

[117] L. Visinelli and J. Redondo, Axion miniclusters in modified cosmological histories, Phys. Rev. D 101, 023008 (2020).

[118] N. Blinov, M. J. Dolan, and P. Draper, Imprints of the early Universe on axion dark matter substructure, Phys. Rev. D 101, 035002 (2020).

[119] E. W. Kolb and I. I. Tkachev, Femtolensing and picolensing by axion miniclusters, Astrophys. J. Lett. 460, L25 (1996).

[120] K. M. Zurek, C. J. Hogan, and T. R. Quinn, Astrophysical effects of scalar dark matter miniclusters, Phys. Rev. D 75, 043511 (2007).

[121] M. Fairbairn, D. J. E. Marsh, and J. Quevillon, Searching for the QCD Axion with Gravitational Microlensing, Phys. Rev. Lett. 119, 021101 (2017).

[122] M. Fairbairn, D. J. E. Marsh, J. Quevillon, and S. Rozier, Structure formation and microlensing with axion miniclusters, Phys. Rev. D 97, 083502 (2018).

[123] S. Borsanyi et al., Calculation of the axion mass based on high-temperature lattice quantum chromodynamics, Nature (London) 539, 69 (2016).

[124] M. Venegas, Relic density of axion dark matter in standard and non-standard cosmological scenarios, arXiv:2106 .07796 .

[125] P. Arias, N. Bernal, D. Karamitros, C. Maldonado, L. Roszkowski, and M. Venegas, New opportunities for axion dark matter searches in nonstandard cosmological models, J. Cosmol. Astropart. Phys. 11 (2021) 003.

[126] D. Hooper, G. Krnjaic, J. March-Russell, S. D. McDermott, and R. Petrossian-Byrne, Hot gravitons and gravitational waves from kerr black holes in the early universe, arXiv:2004.00618. 
[127] A. G. Riess et al., A $2.4 \%$ determination of the local value of the Hubble Constant, Astrophys. J. 826, 56 (2016).

[128] M. Escudero, D. Hooper, G. Krnjaic, and M. Pierre, Cosmology with a very light $\mathrm{L}_{\mu}-\mathrm{L}_{\tau}$ gauge boson, J. High Energy Phys. 03 (2019) 071.
[129] S. Vagnozzi, New physics in light of the $H_{0}$ tension: An alternative view, Phys. Rev. D 102, 023518 (2020).

[130] J. Alcaniz, N. Bernal, A. Masiero, and F. S. Queiroz, Light dark matter: A common solution to the lithium and $H_{0}$ problems, Phys. Lett. B 812, 136008 (2021). 